\section{Robinson et al. (1989) deserves another look}

\section{MICHELA GALLAGHER University of North Carolina, Chapel Hill, North Carolina}

According to Keith and Rudy in this issue of Psychobiology, our view that the behavioral impairments induced by MK-801 support an NMDA-memory hypothesis is "gratuitous," and our results should be dismissed. Let me be equally blunt. The commentary on our work by Keith and Rudy is flawed and misleading. They omit inconvenient data and distort the material they choose to discuss. Of necessity, then, part of my response must correct these errors. In addition, I will raise the question of whether the basic premise they use to criticize our research is even reasonable.

Keith and Rudy suggest that the behavioral impairments produced by MK-801 in our experiments (Robinson, Crooks, Shinkman, \& Gallagher, 1989) merely reflect nonspecific effects on sensory and motor function. In support of their position, they cite a study of the effects of MK-801 on spatial learning in the water maze, in which behavioral abnormalities were reported (Mondadori, Weiskrantz, Buerki, Petschke, \& Fagg, 1989). They do not mention the fact that the lowest dose used by Mondadori et al. in the spatial learning task was higher than any dose used in our study (not to mention the fact that this assessment was conducted with another speciesgerbils). Although it is of doubtful relevance to our work (with rats), those investigators actually state that doses of MK-801 below $0.1 \mathrm{mg} / \mathrm{kg}$ given to mice in other tests "were without obvious behavioral effects" (Mondadori et al., 1989, p. 454). The doses used in our study ranged from 0.01 to $0.08 \mathrm{mg} / \mathrm{kg}$.

In our paper, we acknowledge that high doses of MK-801 produce gross behavioral disturbances in rats, and we warn against the use of such doses. Our data show, however, that a prominent spatial learning impairment is evident at a dose $(0.05 \mathrm{mg} / \mathrm{kg})$ that has no detectable effect on relevant performance indices. In the water maze, this dose impaired spatial learning by all measures assessed (path lengths and latencies on training trials, as well as spatial bias on a free swim with the platform removed). This deficit produced by MK-801 on spatial learning will soon be confirmed in reports from other laboratories (Shapiro \& Caramanos, 1989, in press; Tilson, personal communication). Unacknowledged by Keith and Rudy, in our study we also reported that the $0.05-\mathrm{mg} / \mathrm{kg}$ dose of MK-801 had no detectable effect either on swimming performance during habituation in the maze prior to train-

Correspondence may be addressed to Michela Gallagher, Department of Psychology, Campus Box 3270, Davie Hall, University of North Carolina, Chapel Hill, NC 27599. ing or on escape latencies during cue training when a visible platform was used. Incidentally, the same basic protocol (intertrial interval length, etc.) was used in each version of the task, spatial and cue learning. Finally, animals trained under the drug had persistent deficits when tested on a free swim in the absence of the drug. In contrast, introduction of a $0.05-\mathrm{mg} / \mathrm{kg}$ dose prior to a free swim after the completion of training did not disrupt accurate performance. These data are all relevant to the issue of whether or not MK-801 merely interferes with sensorimotor function, and they provide evidence that this was not the case at a dose that impaired accurate learning.

In another behavioral task in the same study, we found that MK-801 disrupted acquisition in taste potentiation of odor aversion learning. This observation is mentioned by Keith and Rudy, but the careful attention in our experiments to the issue of learning versus sensorimotor impairments is not acknowledged in their critique. In this task, an animal learns an aversion to an odor that is paired with delayed illness only if the odor is presented in compound with a taste. In our paper, we reported that the dose of MK-801 that impaired this form of learning $(0.05 \mathrm{mg} / \mathrm{kg})$ had no effect on baseline drinking, the behavioral response used to measure learning. Thus a drug-induced change in motor capacity or a motivational state relevant to the training and testing conditions is unlikely. More importantly, at this dose MK-801 did not affect the acquisition of simple conditioned responses when either the taste or the odor alone was paired with an effective unconditioned stimulus - that is, with illness in the case of the taste cue, and shock in the case of the odor cue. These results provide relevant and appropriate control for drug-induced changes in the processing of the elements in the learning task (stimuli used as CSs and USs and response measures). These data were not mentioned in the Keith and Rudy commentary.

In their reply to our rebuttal (should they choose to make one), Keith and Rudy might still find some other fault with our attempts to determine whether or not the behavioral impairments we found are due to performance deficits. Of course, ideal control conditions are difficult to obtain, and the philosophical issue of separating learning from performance is problematic indeed. But our considerable efforts to ensure that intact sensorimotor capacities were evident in our animals that had deficits in acquisition of learning are ignored in their critique. Instead, an irrelevant study is cited to discredit our work.

Of course the question of learning versus performance is not the central issue in Keith and Rudy's objection to our work. Our findings are not relevant to the NMDAmemory hypothesis, they claim, because MK-801 does not block LTP under conditions appropriate for comparison with our studies - that is, in vivo induction of LTP. In the paper they cite, it is concluded that "MK-801 clearly [is] effective in blocking LTP induction via a 
peripheral route of administration"' (Abraham \& Mason, 1988 , p. 45). It is true that the dose that blocked LTP was higher than the doses we used, and that the time course for this drug effect on in vivo LTP was different from the testing parameters used in our study. Keith and Rudy fail, however, to mention that the work by Abraham and Mason (1988) was done with anesthetized animals. This was also the case for the work described in an abstract by Halliwell and Morris (1987). In a more recent study, it has been reported that MK-801 antagonizes in vivo LTP in unanesthetized rats at even the lowest dose tested $(0.1 \mathrm{mg} / \mathrm{kg}$; Gilbert \& Mack, 1989, 1990).

These new data aside, Keith and Rudy argue that the effect of MK-801 on LTP observed by Abraham and Mason (1988) is not meaningful because it was obtained at a dose that diminished the population spike response to test pulse stimulation of the perforant path. Abraham and Mason attempted to control for this in their work and concluded that when the population spike was restored to normal size prior to tetanization, a "near total prevention of LTP (4\% increase) still occurred for the MK-801 group" (p. 44). In any event, other published work indicates that the effect of noncompetitive NMDA antagonists on LTP can be dissociated from their effects on responses to test stimulation. Stringer and Guyenet (1983) showed that the effect of systemic phencyclidine administration on the population spike could be eliminated by midbrain transection without altering the blockade of LTP by this drug. These earlier findings are fully discussed in the Abraham and Mason (1988) paper. Furthermore, the recent work by Gilbert and Mack (1990) indicates that, in the unanesthetized animal, MK-801 interferes with LTP without producing any discernible change in the population spike response to test stimuli.

To summarize this part of our rebuttal to Keith and Rudy: It is apparent that their criticisms are based on a selective presentation of the available findings and on a misrepresentation of our data. We do not think that the effects of MK-801, in our hands, merely reflect nonspecific performance deficits. Furthermore, there is no justification for dismissing our data on the basis of work that may not be directly relevant to the conditions of our experiments (comparison of doses in anesthetized and awake animals). The flaws of Keith and Rudy's critique can thus be revealed by an unbiased examination of the available data. On to a more debatable issue!

Keith and Rudy suggest that the relevance of any effects of NMDA antagonists on learning and memory can be disputed if the specific pharmacological parameters used in such behavioral work are not capable of blocking LTP. For example, there should be no mismatch in the effective doses for producing behavioral impairment and for blocking LTP. This criterion might be acceptable if electrically induced LTP actually mimicked the physiological pattern of activity during learning. However, it seems unlikely that stimulation with an electrode in a pathway containing a high density of afferents resembles the neural pattern of information processing in a complex sys- tem. Why, then, should the same drug doses work in these two different situations? Particularly in the case of noncompetitive NMDA antagonists, which are thought to block only open channels, the pharmacological requirement for bioavailability of the drug might depend on the numbers of active sites and their pattern/density within a given region.

A more appropriate test of the NMDA-memory hypothesis may eventually be carried out by comparing the behavioral effects of NMDA antagonists with their effects on experience-dependent forms of potentiation in hippocampus such as those observed in a number of studies (Green \& Greenough, 1986; Sharp, McNaughton, \& Barnes, 1985; Skelton, Scarth, Wilkie, Miller, \& Phillips, 1987). Presumably, the induction of potentiation by experience and/or training procedures should more closely resemble the conditions of the behavioral studies now in question. In the absence of such data, we suggest that the general characteristics of NMDA-dependent LTP provide the most appropriate criteria for assessing the relevance of behavioral impairments induced by NMDA antagonists to this form of neural plasticity. Thus, NMDA antagonists that interfere with acquisition of learning should not interfere with performance of previously acquired learning, because an NMDA mechanism appears to be critical for induction, but not maintenance, of LTP (Cotman \& Monaghan, 1988). In addition, the pharmacological characterization of behavioral effects with these agents is extremely important, because all drugs have nonspecific properties. Thus it is relevant that both competitive and noncompetitive NMDA antagonists (which act at different sites to interfere with NMDA function) produce comparable impairment, as, for example, in the acquisition of spatial learning and taste-odor potentiation (Crooks, Robinson, Hatfield, Graham, \& Gallagher, 1989; Morris, Anderson, Lynch, \& Baudry, 1986; Robinson et al., 1989). In addition, each of these forms of learning has also exhibited stereospecificity in comparisons of $(+)$ and $(-)$ isomers of AP-5 (Crooks et al., 1989; Morris et al., 1986).

Finally, it is my impression that none of the investigators targeted in this critique have promoted the interpretations imputed to them by Keith and Rudy. This is evident in the passages quoted from each of our studies in their paper. As cited by them, we said that our results "are consistent with the view that an NMDA mechanism is critical for certain forms of learning" (emphasis added). Probably not all NMDA sites in the nervous system are associated with LTP. Thus we do not know whether the behavioral effects of NMDA antagonists are at sites that subserve this form of plasticity. However, our results are not inconsistent with the concept that an LTP-like process, engaged by training and dependent on intact NMDA function, is the target of our treatments. Is it improper, then, to be so bold as Morris in saying that these data "add support to the hypothesis that LTP is involved in some, but not all, forms of learning" (Morris et al., 1986, p. 774)? No stronger claim than this has been made by 
us. Indeed, the major theme of our paper, as indicated by its title, is simply that the impairments produced by MK-801 resemble the effects of hippocampal damage.

The role of the NMDA receptor in the induction of LTP is, in my view, one of the most exciting discoveries in the field of neurobiology in recent years. The drugs that are currently available for examination of the functional significance of the NMDA site for the storage of information in intact behaving animals are potentially powerful tools for research. They are also powerful compounds, with steep dose-response functions and many undesirable effects at higher doses. Research using these drugs should be thoughtfully conducted and subject to careful scrutiny. The commentary by Keith and Rudy highlights some of the important issues that deserve concern. Nonetheless, I assert that the case they make against the significance of our work (that MK-801 does not block LTP and that our behavioral deficits are due to sensorimotor impairment) is simply not supported by the evidence, most of which was either omitted or distorted in their critique. Although public forums for debate are essential for a healthy and lively scientific atmosphere, such debates should be conducted on firmer ground with a fair representation of the pertinent data. As it stands, I am unwilling to reject the NMDA-memory hypothesis, and I find some evidence, albeit not definitive, to support it.

\section{REFERENCES}

abraham, W. C., \& Mason, S. E. (1988). Effects of the NMDA receptor/channel antagonists $\mathrm{CPP}$ and MK801 on hippocampal field potentials and long-term potentiation in anesthetized rats. Brain Research, 462, 40-46.

Cotman, C. W., \& Monaghan, D. T. (1988). Excitatory amino acid neurotransmission: Receptors and Hebb-type synaptic plasticity. Annual Review of Neuroscience, 11, 61-80.

Crooks, G. B., Robinson, G. S., Hatfield, T. J., Graham, P. W.,
\& Gallagher, M. (1989). Intraventricular administration of the NMDA antagonist APV disrupts learning of an odor aversion that is potentiated by taste. Society for Neuroscience Abstracts, 15, 464.

GILBERT, M. E., \& MACK, C. M. (1989). Blockade of LTP, kindling, and kindling-induced potentiation by the NMDA-antagonist, MK-801 . Society for Neuroscience Abstracts, 15, 1101.

GiLberT, M. E. , MACK, C. M. (1990). The NMDA antagonist MK801 suppresses long-term potentiation, kindling, and kindling-induced potentiation in the perforant path of the unanesthetized rat. Brain Research, 519, 89-96.

Green, E. J., \& Greenough, W. T. (1986). Altered synaptic transmission in dentate gyrus of rats reared in complex environments: Evidence from hippocampal slices maintained in vitro. Journal of Neurophysiology, 55, 739-750.

HALLIWELl, R. F., \& MoRRIS, R. G. M. (1987). Anticonvulsant doses of MK-801 fail to block long-term potentiation in vivo, or impair spatial memory in the rat. Neuroscience Letters Supplement, 29, 599.

Mondadori, C., Weiskrantz, L., Buerki, H., Petschke, F., \& FagG, G. E. (1989). NMDA receptor antagonists can enhance or impair learning performance in animals. Experimental Brain Research, 75, 449-456.

Morris, R. G. M., ANderson, E., Lynch, G. S., ANd Baudry, M. (1986). Selective impairment of learning and blockade of long-term potentiation by an $N$-methyl-D-aspartate receptor antagonist, AP5. Nature, 319, 774-776.

Robinson, G. S., JR., Crooks, G. B., Jr., Shinkman, P. G., \& GALLAGHER, M. (1989). Behavioral effects of MK-801 mimic deficits associated with hippocampal damage. Psychobiology, 17, 156-164.

Shapiro, M. L., \& Caramanos, Z. (1989). NMDA antagonist MK-801 impairs acquisition but not performance of spatial working and reference memory. Society for Neuroscience Abstracts, 15, 464.

Shapiro, M. L., \& Caramanos, Z. (1990). NMDA antagonist MK-801 impairs acquisition but not performance of spatial working and reference memory. Psychobiology, 18, 231-243.

Sharp, P. E., McNaughton, B. L., Barnes, C. A. (1985). Enhancement of hippocampal field potentials in rats exposed to a novel, complex environment. Brain Research, 339, 361-365.

Skelton, R. W., Scarth, A. S., Wilkie, D. M., Miller, J. J., * Phillips, A. G. (1987). Long-term increases in dentate granule cell responsivity accompany operant conditioning. Journal of Neuroscience, 7, 3081-3087.

Stringer, J. L., \& GuYenet, P. G. (1983). Elimination of long-term potentiation in the hippocampus by phencyclidine and ketamine. Brain Research, 258, 159-164. 